\title{
Does Integrated Fixation Provide Benefit in the Reconstruction of Posttraumatic Tibial Bone Defects?
}

\author{
Mitchell Bernstein MD, Austin T. Fragomen MD, Samir Sabharwal BA, \\ Jonathan Barclay BA, S. Robert Rozbruch MD
}

Published online: 5 May 2015

(C) The Association of Bone and Joint Surgeons (B) 2015

\begin{abstract}
Background Limb salvage in the presence of posttraumatic tibial bone loss can be accomplished using the traditional Ilizarov method of distraction osteogenesis with circular external fixation. Internal fixation placed at the beginning of the consolidation phase, so-called integrated fixation, may allow for earlier removal of the external
\end{abstract}

One of the authors (ATF) lists the following relevant financial activities outside of this work and/or any other relationships or activities that readers could perceive to have influenced, or that give the appearance of potentially influencing, this manuscript: consultant for Smith \& Nephew, Inc (Memphis, TN, USA), and Synthes (Paoli, PA, USA) in the amount of less than USD 10,000. One of the authors (SRR) lists the following relevant financial activities outside of this work and/or any other relationships or activities that readers could perceive to have influenced, or that give the appearance of potentially influencing, this manuscript: Smith \& Nephew, Inc in the amount of less than USD 10,000.

All ICMJE Conflict of Interest Forms for authors and Clinical Orthopaedics and Related Research ${ }^{\circledR}$ editors and board members are on file with the publication and can be viewed on request.

Clinical Orthopaedics and Related Research ${ }^{\circledR}$ neither advocates nor endorses the use of any treatment, drug, or device. Readers are encouraged to always seek additional information, including FDAapproval status, of any drug or device prior to clinical use.

Each author certifies that his or her institution approved the human protocol for this investigation, that all investigations were conducted in conformity with ethical principles of research, and that informed consent for participation in the study was obtained.

M. Bernstein $(\square)$

Department of Orthopaedic Surgery \& Rehabilitation, Stritch

School of Medicine, Loyola University Medical Center,

2160 S First Avenue, Maywood, IL 60153, USA

e-mail: mitchell.bernstein@lumc.edu

A. T. Fragomen, S. R. Rozbruch

Limb Lengthening and Complex Reconstruction Service,

Hospital for Special Surgery, New York, NY, USA fixator but introduces concerns about cross-contamination from the additional open procedure and maintenance of bone regenerate stability.

Questions/purposes Among patients deemed eligible for integrated fixation, we sought to determine: (1) Does integrated fixation decrease the time in the external fixator? (2) Is there a difference in the rate of complications between the two groups? (3) Are there differences in functional and radiographic results between integrated fixation and the traditional Ilizarov approach of external fixation alone?

Methods Between January 2006 and December 2012, we treated 58 patients (58 tibiae) with posttraumatic tibial bone loss using the Ilizarov method. Of those, 30 patients $(52 \%)$ were treated with the "classic technique" (external fixator alone) and $28(48 \%)$ were treated with the "integrated technique" (a combination of an external fixator and plating or insertion of an intramedullary nail). During that period, the general indications for use of the integrated technique were closed physes, no active infection, and a healed soft tissue envelope located at the intended internal fixation site; the remainder of the patients were treated with the classic technique. Followup on $30(100 \%)$ and 28 $(100 \%)$ patients in the classic and integrated techniques, respectively, was achieved at a minimum of 1 year (mean, 3 years; range, 1-8 years). Adverse events were reported as problems, obstacles, and complications according to the

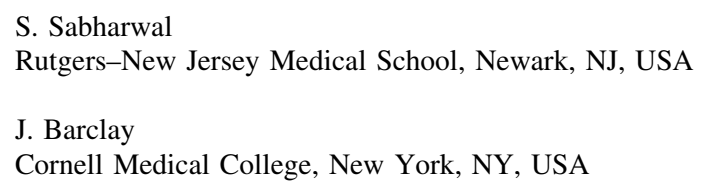


publication by Paley. Problems and obstacles are managed by nonoperative and operative means, respectively; in addition, they resolve completely with treatment. Complications, according to the Paley classification, result in permanent sequelae. Functional and radiographic results were reported using the Association for the Study and Application of Methods of Ilizarov scoring system.

Results Overall, there was a mean of four (range, 2-5) surgical procedures to complete the tibial reconstruction with a similar incidence of unplanned surgical procedures (obstacles) between the two groups $(\mathrm{p}=0.87)$. Patients treated with integrated fixation spent less time in the external fixator, 7 months (range, 5-20 months) versus 11 months (range, $1-15$ months; $\mathrm{p}<0.001$ ). There were seven problems, 15 obstacles, and zero complications in the classic group. Ten problems, 15 obstacles, and one complication occurred in the integrated fixation group. There was no difference in the severity $(p=0.87)$ or number ( $\mathrm{p}=0.40$ ) of complications between both groups. Good to excellent Association for the Study and Application of Methods of Ilizarov function and bone scores were obtained in $100 \%$ and $98 \%$ of patients, respectively.

Conclusions The integrated fixation method allows for a more efficient limb salvage surgical reconstruction in patients carefully selected for that approach, whereas the frequency of adverse events and ability to restore limb lengths was not different between the groups with the numbers available. Careful placement of external fixation pins is critical to decrease cross-contamination with planned internal fixation constructs. In this study of posttraumatic tibial bone defect reconstruction, good/excellent results were found in all patients after a mean of four surgical procedures; however, a larger multicenter prospective study would allow for more robust and generalizable conclusions.

Level of Evidence Level III, therapeutic study.

\section{Introduction}

The Ilizarov principles of distraction osteogenesis using circular frames have made posttraumatic reconstruction of limbs a viable option [18, 34], often obviating the need for an amputation. However, external fixators are cumbersome for patients [13, 26, 31] and have a time-dependent incidence of complications (pin-tract infection, pin breakage, soft tissue irritation, and joint stiffness); therefore, the impetus for decreasing the duration of the external fixator is an important source of intervention. Generally, limb reconstruction is performed through bone transport separated into three phases: (1) latency; (2) distraction; and (3) consolidation. After a 7-day latency period, the distraction phase begins as the segment is lengthened at a typical rate of $1 \mathrm{~mm}$ per day divided into four $0.25-\mathrm{mm}$ increments. The consolidation phase consists of bone regenerate and docking site healing. The consolidation phase is typically twice as long as the distraction phase, making it an effective source of intervention to decrease the time the patient spends in the external fixator. This can be achieved by supplanting the external fixator with internal fixation during, or subsequent to, lengthening and deformity correction, respectively $[1,9,25$, $28,39,41]$. The use of internal fixation with intramedullary nails or plates and screws has been described to stabilize the regenerate allowing for earlier external fixator removal [10, $16,33]$. The advantage of decreased external fixation time is tempered with the potential risks of medullary canal sepsis (supplanted intramedullary nailing), cross-contamination with surface implants, and maintenance of reduction with potentially less rigid constructs [16, 19, 22, 28].

Surgeons using the Ilizarov method for limb reconstruction appreciate the lengthy consolidation phase"lengthening over a nail" and "bone transport over a nail" (Fig. 1) were some of the first modifications that combined the use of internal and external fixation to mitigate the time waiting for bony consolidation and frame removal [20, 22, 28, 40]. The requisites of this procedure are: a straight bone with a large enough canal diameter to accommodate an intramedullary nail and external fixation pins that do not come into contact with each other. Integrated fixation, as defined in this study, evolved as an impetus to increase the efficiency in the limb reconstruction episode of care (application of external fixator, removal of nonviable bone, correcting deformity, achieving bone union, and removal of the external fixator). Lengthening and then insertion of a plate (Fig. 2) [16] and lengthening and then insertion of an intramedullary nail (Fig. 3) [33] were techniques that built on previous published techniques and were modified to circumvent some of the challenges that existed with older techniques such as the inability to use in patients with a preexisting deformity, presence of intramedullary sepsis, and small periarticular bone segments. Posttraumatic tibial bone defects are not common and there is little highquality (Level I) evidence as to the safest and most efficient limb reconstruction method [10, 14, 16, 27]. We compared the integrated fixation technique with the classic group in a uniform group of patients (bone defects of the tibia exclusively as a result from trauma) to elucidate if combining internal and external fixation is a safe manner to expedite the time patients are in an external fixator.

Among patients deemed eligible for integrated fixation, we therefore sought to determine: (1) Does integrated fixation decrease the time in the external fixator? (2) Is there a difference in the rate of complications between the two groups? (3) Are there differences in functional and radiographic results between integrated fixation and the traditional Ilizarov approach of external fixation alone? 


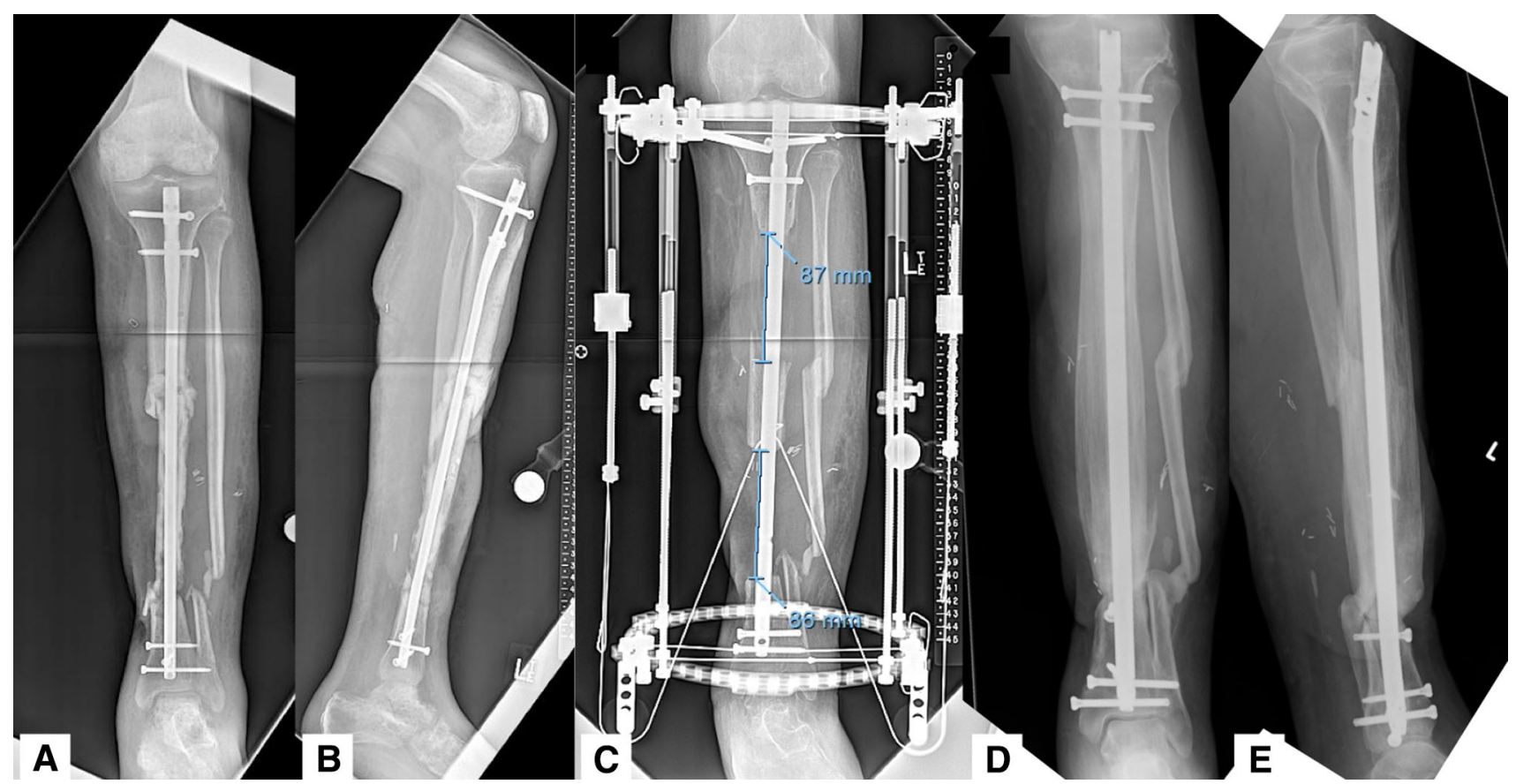

Fig. 1A-E (A) AP and (B) lateral radiographs show a 31-year-old man who presented with a $17.5-\mathrm{cm}$ segment of infected tibial bone after a Type IIIB open tibia fracture. The patient was treated with soft tissue coverage, excision of nonviable bone, and bone transport over a nail (C). Final radiographs (D-E) demonstrate maintenance of alignment, docking site union, and no infection 1 year after reconstruction.

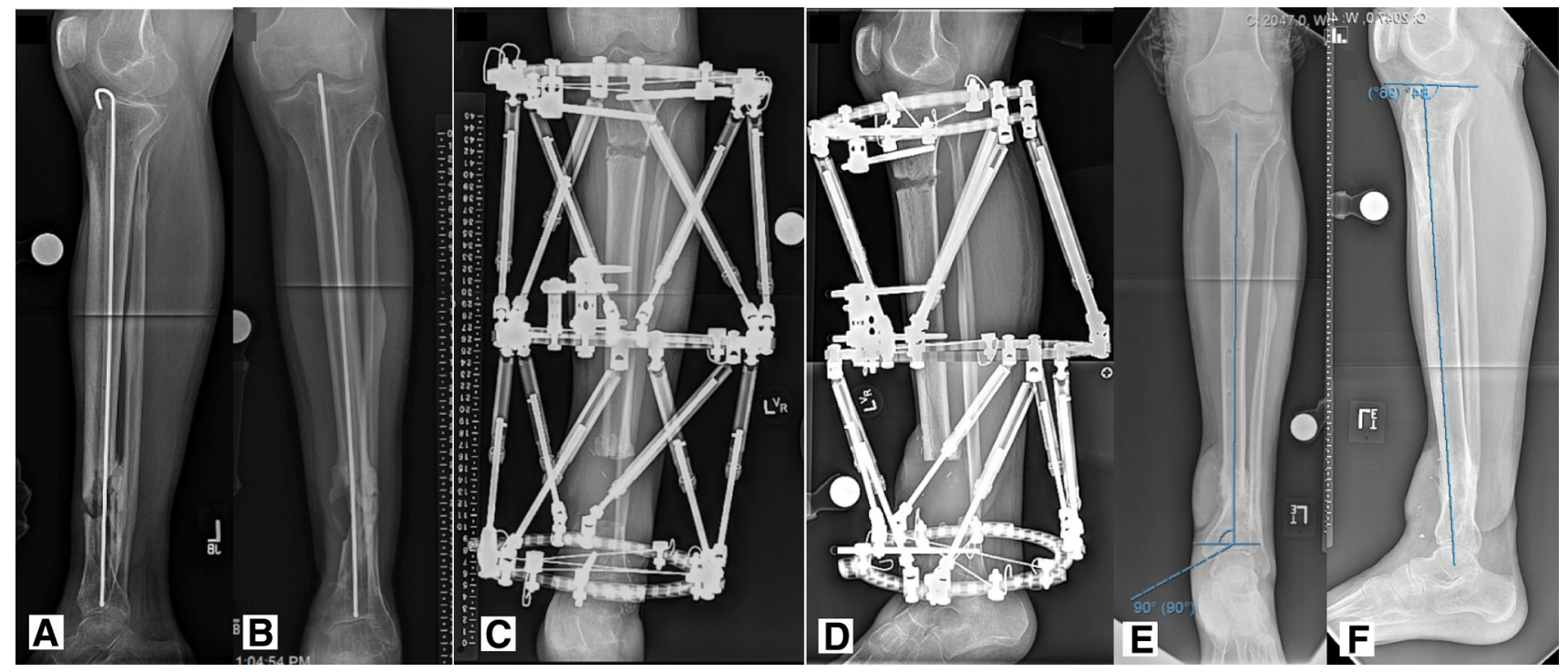

Fig. 2A-F (A) AP and (B) lateral radiographs show a 34-year-old man with a $6-\mathrm{cm}$ distal tibial metaphyseal bone defect. The patient was treated with resection of the nonviable segment of bone, proximal tibia osteotomy, and transport using the "classic method" of

\section{Patients and Methods}

Between January 2006 and December 2012, we treated 58 patients (58 tibiae) with posttraumatic tibial bone loss using the Ilizarov method. These patients were identified retrospectively from our limb lengthening and deformity distraction osteogenesis $(\mathbf{C}-\mathbf{D})$. Final radiographs $(\mathbf{E}-\mathbf{F})$ demonstrate restoration of limb lengths and normal coronal and sagittal alignment. The patient was in the external fixator for 302 days.

database. Of those, 30 patients $(52 \%)$ were treated with the "classic technique" (external fixator alone) and 28 (48\%) were treated with the "integrated technique" (a combination of an external fixator and plating or insertion of an intramedullary nail). One patient had treatment of bone transport over a nail. During that period, the general 


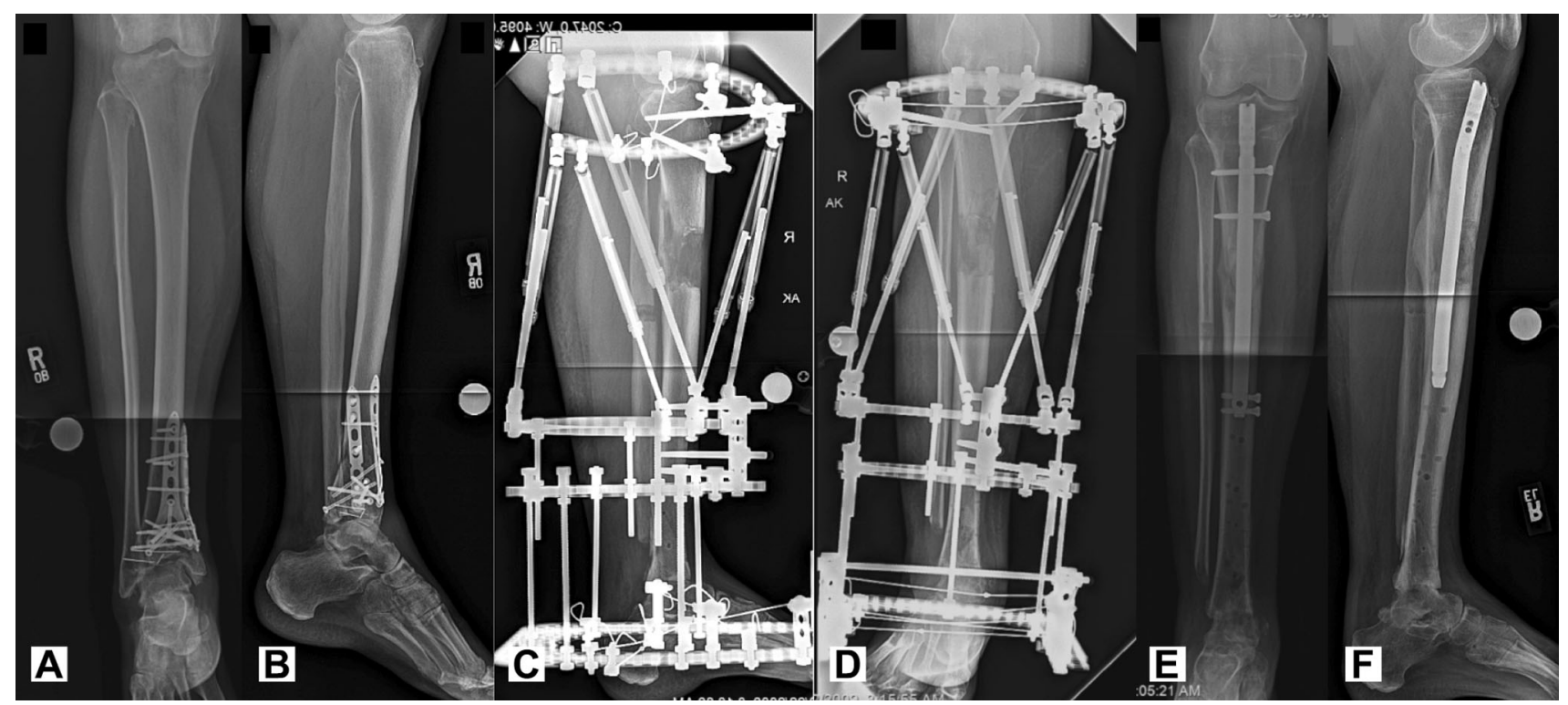

Fig. 3A-F (A) AP and (B) lateral radiographs show a 50-year-old woman with an infected pilon fracture and $4 \mathrm{~cm}$ of nonviable bone at the ankle. The patient was treated with "integrated fixation,"

indications for use of the integrated technique were closed physes, no active infection, and healed soft tissue envelope located at the intended internal fixation site; the remainder of the patients were treated with the classic technique. Followup on $30(100 \%)$ and $28(100 \%)$ patients in the classic and integrated techniques, respectively, was achieved at a minimum of 1 year (mean, 3 years; range, 1-8 years). Adverse events were reported as problems, obstacles, and complications according to the publication by Paley. Problems and obstacles are managed by nonoperative and operative means, respectively; in addition, they resolve completely with treatment. Complications, according to the Paley classification, result in permanent sequelae.

Functional and radiographic results were reported using the Association for the Study and Application of Methods of Ilizarov scoring system [27]. Functional results can be classified as excellent, good, fair, poor, or failure. It is based on five criteria: activity level, ambulation status (limp), joint stiffness (loss of $15^{\circ}$ of motion), presence of complex regional pain syndrome, and pain level. An excellent result is an active patient with none of the latter four criteria. A good result is an active individual with two or less of the latter four criteria. A fair result is one who is active with three or all of the latter criteria. A poor result is an inactive patient (unemployed, inability to perform activities of daily living as a result of the injury); failure is defined as a patient who requires an amputation. The bone results are classified as excellent, good, fair, or poor based on four criteria. These are: union, presence of infection, lengthening $(\mathbf{C}-\mathbf{D})$ and then insertion of an intramedullary nail $(\mathbf{E}-\mathbf{F})$. The patient was in the external fixator for 183 days.

deformity (less than $7^{\circ}$ ), and limb length discrepancy (less than $2.5 \mathrm{~cm}$ ). An excellent bone result is a patient who has a union, no infection, deformity, or limb length discrepancy. A good result is union and absence of two or more of the latter criteria (infection, deformity, limb length discrepancy). Fair results occur in patients with union and one of the following: absence of infection, deformity, or limb length discrepancy. Poor is classified as a patient with an infected nonunion with deformity and limb length discrepancy (greater than $2.5 \mathrm{~cm}$ ).

Institutional review board approval was obtained before initiation of the study. Surgical procedures were performed at one institution, a quaternary orthopaedic referral center, by one of two fellowship-trained surgeons (ATF, SRR) in limb lengthening and complex extremity reconstruction. The study was chosen to begin in 2006 because this was the commencement of the picture archiving and communication system in our institution and would help minimize any patient exclusion resulting from misplaced radiographs.

The patients were captured by 2014 Current Procedural Terminology (CPT) codes. The Limb Lengthening and Complex Reconstruction Service database was searched using CPT codes that would generate an exhaustive list of potentially applicable patients: 27720 (repair of nonunion or malunion, tibia; without graft); 27724 (repair of nonunion or malunion, tibia; with iliac or other autograft); 27745 (prophylactic treatment, nailing, pinning, plating, or wiring, tibia); 27715 (osteotomy or osteoplasty tibia); 27870 (ankle fusion); 27724 (tibial nonunion); and 27640 (resection tibia). Because this list would contain patients 
not applicable for the study, a second list was created using combined CPT codes: (27715/27870, 27715/27724, 27715/27720, 27745/277870, 27745/27720, 27745/27724).

The list was screened for patient duplications, generating a third list containing 177 patients.

From January 2006 through December 2012, the charts of the 177 patients were reviewed to elucidate the etiology and location of bone defect. Patients without isolated bone loss of the tibia that resulted from trauma were excluded. Fifty-eight patients (58 tibiae) with posttraumatic tibia bone loss comprised the final list of patients; 30 patients $(52 \%)$ were treated with the "classic technique" (external fixator alone) and 28 (48\%) were treated with the "integrated technique." During the first half of the study period, (January 2006 through May 2009), 13 patients (48\%) underwent classic reconstruction and 14 patients (52\%) were treated with integrated fixation (eight lengthening and then plating procedures, six lengthening and then nailing procedures). During the second half of the study period, (June 2009 through December 2012), 17 patients (55\%) were treated with the classic technique and 14 patients (45\%) with the integrated technique (three lengthening and then plating procedures, 10 lengthening and then nailing procedures, and one bone transport over a nail procedure). The prolonged use of external fixation is arduous for the patient and has a time-dependent incidence of complications; the limb salvage pathway using Ilizarov methods has evolved over the study period to find a safe way to use internal fixation to eliminate time the patient spends in the external fixator during the consolidation phase. The decision to use less lengthening and then the plating technique during the second half of the study emerged from our experience of regenerate bending with the plate [16] and the availability of custom (short) intramedullary nails in the lengthening and then nailing group.

The location of the bone defects varied among patients. Thirty-nine patients had distal tibial bone defects, 10 patients had diaphyseal defects, and the remaining eight patients had proximal defects. In the classic group, 20 were distal defects, five were diaphyseal, and five were proximal. In the integrated fixation group, 20 were distal defects, five were diaphyseal, and three were proximal. Demographic and surgical variables were collected retrospectively from chart review and radiographs. No patients were lost to followup. Twenty patients $(34 \%)$ had not been seen within the preceding 2 years of the data collection for this study. Eleven patients $(55 \%)$ were treated in the classic group and nine $(45 \%)$ in the integrated fixation group (five lengthening and then plating procedures and four lengthening and then nailing procedures).

Limb reconstruction in this patient population is challenging and requires a staged approach. Tibial pathology is addressed using the débridement principles of Cierny and Mader [4]. Débridement of the nonunion site is performed, all nonviable bone is resected, and previous hardware, if present, is removed. Bone viability is determined by observing bleeding after drilling with a $1.8-\mathrm{mm}$ Ilizarov wire or 2.0-mm drill bit. Five deep intraoperative cultures are obtained. Application of the circular external fixator is used for stability and eventual limb reconstruction. If present, antibiotic beads are used for dead space management; otherwise, gradual compression (shortening) of the defect is performed. Twenty-three patients (40\%) had acute dead space management, and the specific name for the limb reconstruction is termed "shortening-distraction." The dead space is closed as quickly as the vascular status can tolerate (ie, without kinking of the blood vessels). This is at a rate of $2 \mathrm{~mm}$ per day. The mean defect size in this group was $41 \mathrm{~mm}$ (range, 16-84 mm). Thirtyfive patients $(60 \%)$ had staged dead space management. The average defect size in this group was $59 \mathrm{~mm}$ (range, $16-130 \mathrm{~mm})$.

If culture-positive, appropriate intravenous antibiotics are administered by an infectious disease specialist. Soft tissue lesions (chronic draining sinuses, exposed bone, invaginated skin) are managed with a plastic surgeon experienced in limb reconstruction using local, rotational, and free flaps as soon as the débridement is completed.

Patients are given a brochure with clinical photographs on how to recognize a pin site infection (redness, drainage, new pain). They are instructed to contact our office if they suspect an infection and commence a 10-day course of $500 \mathrm{mg}$ cephalexin orally, four times daily. If penicillin-allergic, or suspicion of methicillin-resistant Staphylococcus aureus infection, 160/800 mg trimethoprim/sulfamethoxazole orally twice daily is given.

Staged osteoplasty of the tibia for lengthening is typically performed 6 weeks later. This staged treatment occurred in 35 patients $(60 \%)$. Insertion of internal fixation occurs once the deformity correction is completed. This usually coincides with nonunion site healing and thus the entire tibial frame can be removed.

Two groups of patients were compared based on the surgical treatment, termed the "classic technique" (Fig. 2) or "integrated technique" (Fig. 3). If a patient's deformity correction and lengthening were performed solely using an external fixator, it was termed the "classic technique." Those patients who underwent deformity correction with an external fixator and then application of a plate, or insertion of an intramedullary nail, were termed the "integrated technique." In the integrated group, 16 patients underwent lengthening and then nailing, 11 patients underwent lengthening and then plating, and one patient underwent bone transport over a nail. The external fixator used in all patients was a Taylor Spatial Frame (Smith \& Nephew, Memphis, TN, USA), which is FDA-approved. 
The "rings first" method was used to mount the frame to the tibia in all cases [16, 32]. Mounting parameters were obtained intraoperatively [12]. Six-millimeter tapered hydroxyapatite half-pins (Biomet, Warsaw, IN, USA) and 1.8-mm bayonet-tipped Ilizarov wires were used for fixation. The technique for mounting a frame for a lengthening and then nailing or lengthening and then plating procedure was performed according to Rozbruch et al [33] and Harbacheuski et al [16], respectively. In the lengthening and then nailing group, it is critical to ensure that the external fixator half-pins and wires do not cross the intended path of the staged intramedullary nail.

The postoperative protocol was identical in both patient groups. Patients were allowed to bear weight as tolerated using a walker or crutches on postoperative Day 1 after the external fixator was applied. Supervised physical therapy consisting of knee and ankle ROM occurred three times per week. Patients were also given a daily 1-hour home program. Occupational therapy provided patients with a custom-made foot-resting splint that attached to the external fixator to ensure neutral ankle dorsiflexion.

Fourteen days of deep vein thrombosis prophylaxis using a low-molecular-weight heparin began on postoperative Day 2. The sterile operative dressings were removed on postoperative Day 2 as daily pin care, using one-half peroxide and one half normal saline, was started.

The latency phase began when the low-energy tibial osteotomy was performed. This was delayed approximately 6 weeks in 35 patients $(60 \%)$. The distraction phase began 7 days subsequent to the latency phase. Thus, on postoperative Day 7, after the tibial osteoplasty was performed, distraction occurred at a rate of $1 \mathrm{~mm}$ /day three times per day. The patients performed their own strut adjustments.

During deformity correction with the external fixator, both patient groups had identical followup protocols. During the distraction phase, they were seen in the clinic every 14 days by one of the senior authors (ATF, SRR). Clinical examination involved inspection of the external fixator for stability, pin sites for infection, and wounds for healing. Knee and ankle ROM was recorded using a goniometer. Calibrated orthogonal weightbearing images were assessed for the quality of the regenerate. The AP and lateral distraction gap were measured and correlated with the anticipated lengthening desired in the Taylor Spatial Frame program. Hip-to-ankle radiographs, used to measure mechanical axis deviation, and limb length discrepancy (LLD), were taken once the deformity programs were completed.

For patients in the integrated fixation group, insertion of an intramedullary nail (Synthes, Paoli, PA, USA, and EBI/ Biomet, Warsaw, IN, USA), FDA-approved, or application of a locking plate (Smith \& Nephew), FDA-approved, was performed once the desired alignment was achieved.
Patients were then transitioned to $30 \mathrm{lbs}$ toe-touch weightbearing and only advanced to full weightbearing once three of four cortices demonstrated a bridging callus. This typically occurred over a 6-week period. The followup occurred at 2 weeks for a wound check and in 4- to 6-week intervals with clinical and radiological examinations as those described previously.

Both integrated fixation techniques evolved with the desire to allow for earlier removal of the external fixator. The indications for the lengthening and then plating technique included an apex of deformity in the proximal or distal metaphysis necessitating an osteotomy that would not allow for adequate fixation with an intramedullary nail. In addition, patients with tibial medullary canals that were too small to accommodate an intramedullary nail were chosen for a lengthening and then plating technique. Contraindications included a history of chronic osteomyelitis and a poor soft tissue envelope at the intended plate insertion site [16]. The lengthening and then nailing technique was chosen in patients with closed physes, a large enough canal diameter, and no active infection located at the intended intramedullary nail path [33]. The ability to use a short custom tibial nail later expanded indications for using the lengthening and then nailing over the lengthening and then plating technique.

Baseline demographics, surgical variables, and outcomes were compared. At initial consultation, calibrated 36-inch orthogonal radiographs of the tibia were obtained for coronal and sagittal alignment measurements. A standing 51-inch hip-to-ankle radiograph was also obtained, which allowed for measurement of the mechanical axis deviation and LLD.

Adverse events were reported as problems, obstacles, or complications as described by Paley [26]. This classification was developed to illustrate and qualify some of the unique challenges that occur during deformity correction and lengthening surgery. In addition, it is an attempt to standardize the reported adverse events among the literature. In this classification, problems represent difficulties that arise during treatment that do not require an operative intervention and resolve completely at the end of treatment. An obstacle is an adverse event during treatment that requires surgical intervention, which does cause any permanent disability. A complication is an adverse event that occurs during treatment that does not resolve (eg, foot-drop from a common peroneal nerve injury).

Functional and radiographic results were reported using the Association for the Study and Application of Methods of Ilizarov scoring system [27].

Descriptive statistics presented for this study are means and standard deviations for continuous variables and frequency counts and percentages for categorical variables. Univariate analyses were performed to evaluate the association between method of bone transport and patient and clinical variables of interest. The Shapiro-Wilk test 
Table 1. Demographics*

\begin{tabular}{|c|c|c|c|c|}
\hline \multirow[t]{2}{*}{ Parameter } & \multirow{2}{*}{ Overall $(\mathrm{n}=58)$} & \multicolumn{2}{|c|}{ Method of bone transport } & \multirow[t]{2}{*}{$\mathrm{p}$ value } \\
\hline & & Classic $(\mathrm{n}=30)$ & Integrated $(\mathrm{n}=28)$ & \\
\hline Age (years) & $45(19-61)$ & $43(25-56)$ & $48(19-61)$ & 0.009 \\
\hline Male:female & $39: 19$ & $24: 6$ & $15: 13$ & 0.0497 \\
\hline Infected (culture-positive) & $50 \%$ & $53 \%$ & $46 \%$ & 0.5999 \\
\hline Soft tissue flap present & $29 \%$ & $43 \%$ & $14 \%$ & 0.0195 \\
\hline Smoker & $17 \%$ & $23 \%$ & $11 \%$ & 0.3007 \\
\hline Followup (months) & $33(6-90)$ & $31(6-88)$ & $36(6-90)$ & 0.388 \\
\hline Preoperative LLD (mm) & $35(0-120)$ & $44(0-120)$ & $27(0-70)$ & 0.096 \\
\hline Postdébridement defect size (mm) & $18(0-70)$ & $15(0-60)$ & $21(0-70)$ & 0.171 \\
\hline Total bone loss (mm) & $53(16-130)$ & $57(16-120)$ & $49(16-130)$ & 0.185 \\
\hline Actual length achieved (mm) & $49(8-110)$ & $53(16-110)$ & $44(8-100)$ & 0.248 \\
\hline Time in external fixator (days) & $281(38-587)$ & $336(136-587)$ & $224(38-452)$ & $<0.001$ \\
\hline Final LLD (mm) & $-3(-30$ to 9$)$ & $-9(-20$ to 9$)$ & $-4(-30$ to 0$)$ & 0.896 \\
\hline
\end{tabular}

* Values recorded as mean (range).

LLD $=$ limb length discrepancy.

indicated the continuous variables such as age and time in the external fixator had nonnormal distributions, so the Wilcoxon rank-sum test was used to analyze continuous variables. Categorical variables were examined using the chi square test. Statistical significance was set at $\mathrm{p}<0.05$. All analyses were performed using SAS Version 9.3 (SAS Institute, Inc, Cary, NC, USA).

Overall, there were 58 patients (58 tibiae) with a mean age of 45 years (range, 19-61 years). Thirty patients were treated in the classic group and 28 in the integrated group (Table 1). Analysis of the baseline demographics revealed that patients in the integrated group were older $(p=0.009$; 48 years; range, 19-61 years versus 43 years; range 25-56 years). Patients in the classic group were more likely to be male ( $\mathrm{p}=0.0497 ; 24$ male versus 15 male) and have a soft tissue flap present $(\mathrm{p}=0.0195 ; 43 \%$ patients versus $14 \%$ ). The presence of an infected defect at presentation, smoking status, and total bone loss were similar in both groups (Table 1).

\section{Results}

Overall, there was a mean of four (range, 2-5) surgical procedures to complete the tibial reconstruction with a similar incidence of unplanned surgical procedures (obstacles $)$ in the classic and integrated fixation groups $(\mathrm{p}=0.87$; 15 versus 16). Patients treated with integrated fixation spent less time in the external fixator, 7 months (range, 5-20 months) versus 11 months (range, 1-15 months; $\mathrm{p}<0.001$ ). There were seven problems, 15 obstacles, and zero complications in the classic group. Ten problems, 16 obstacles, and one complication occurred in the integrated fixation group. There were no differences in the severity ( $p=0.87)$ or number $(p=0.40)$ of complications between both groups. There was no difference in the incidence of unplanned surgical procedures (obstacles) between the classic group and integrated group $(\mathrm{p}=0.2194 ; 15$ versus 16) with the numbers available.

Four cases (two in each group) were missing final outcome scores as a result of missing chart data. Of the remaining 54 patients, there were 47 excellent Association for the Study and Application of Methods of Ilizarov function scores (27 in the classic group and 20 in the integrated group), eight good scores (one in the classic group and seven in the integrated group), and zero poor scores. The distribution of Association for the Study and Application of Methods of Ilizarov bone scores were as follows: 51 excellent (27 in the classic group and 24 in the integrated group), two good (one in the classic group and one in the integrated group), and one poor (zero in the classic group and one in the integrated group). There were no failures in either group. There was no difference in the distribution of Association for the Study and Application of Methods of Ilizarov function $(\mathrm{p}=0.7358)$ or bone $(p=0.4815)$ between the two groups.

The external fixation index, defined as time in the external fixator per centimeter of lengthening [11], was also decreased in the integrated fixation group compared with the classic group, 61 days $/ \mathrm{cm}$ versus 75 days $/ \mathrm{cm}$, respectively $(\mathrm{p}=0.033)$.

Overall, there were 48 adverse events in 30 patients (Table 2). These were categorized as 17 problems, 30 obstacles, and one minor complication (Table 3).

No patients had recurrence of infection and all had bony union at final followup. However, it is worth noting that 
Table 2. Description of adverse events

\begin{tabular}{|c|c|c|c|c|c|c|}
\hline \multirow[t]{3}{*}{ Type } & \multicolumn{2}{|c|}{ Overall $(n=48)$} & \multicolumn{4}{|c|}{ Method of bone transport } \\
\hline & \multirow[b]{2}{*}{ Number } & \multirow[b]{2}{*}{ Percent } & \multicolumn{2}{|c|}{ Classic $(n=22)$} & \multicolumn{2}{|c|}{ Integrated $(n=26)$} \\
\hline & & & Number & Percent & Number & Percent \\
\hline Nonunion & 11 & 23 & 5 & 23 & 6 & 23 \\
\hline Pin infection & 10 & 21 & 5 & 23 & 5 & 19 \\
\hline Malunion & 6 & 13 & 3 & 13 & 3 & 12 \\
\hline Delayed union & 5 & 10 & 2 & 9 & 3 & 12 \\
\hline Equinus contracture & 5 & 10 & 3 & 14 & 2 & 8 \\
\hline Entrapment of overlying skin & 4 & 8 & 2 & 9 & 2 & 8 \\
\hline Refracture & 3 & 6 & 1 & 5 & 2 & 8 \\
\hline Osteomyelitis & 1 & 2 & 0 & 0 & 1 & 4 \\
\hline Failure of fibula to separate & 1 & 2 & 0 & 0 & 1 & 4 \\
\hline Hindfoot arthrosis & 1 & 2 & 0 & 0 & 1 & 4 \\
\hline Septic knee & 1 & 2 & 1 & 5 & 0 & 0 \\
\hline
\end{tabular}

Table 3. Adverse events according to Paley

\begin{tabular}{|c|c|c|c|c|c|c|}
\hline \multirow[t]{3}{*}{ Type } & \multicolumn{2}{|c|}{ Overall $(\mathrm{n}=48)$} & \multicolumn{4}{|c|}{ Method of bone transport } \\
\hline & \multirow[b]{2}{*}{ Number } & \multirow[b]{2}{*}{ Percent } & \multicolumn{2}{|c|}{ Classic $(\mathrm{n}=22)$} & \multicolumn{2}{|c|}{ Integrated $(\mathrm{n}=26)$} \\
\hline & & & Number & Percent & Number & Percent \\
\hline Complication & 1 & 2 & 0 & 0 & 1 & 4 \\
\hline Obstacle & 30 & 63 & 15 & 68 & 15 & 58 \\
\hline Problem & 17 & 35 & 7 & 32 & 10 & 38 \\
\hline
\end{tabular}

one patient, who was initially treated with the integrated technique, needed to be salvaged with a classic Ilizarov reconstruction. This was a 57-year-old man with a IIIB open tibial shaft fracture, who was initially treated with bone transport over a nail. He presented 3 months subsequent to his index procedure with acute osteomyelitis and an abscess. This patient required irrigation and débridement and conversion to bone transport using external fixation alone. The patient had a 7-cm tibial shaft defect. At a final followup of 42 months, the patient was infection-free; he was reported to have excellent Association for the Study and Application of Methods of Ilizarov bone and function scores.

\section{Discussion}

Limb salvage for posttraumatic tibial bone loss occurs in patients who are characterized by host and injury parameters that make reconstruction challenging. Application of an external fixator, which imparts prolonged limb stability, and use of Ilizarov principles allow the surgeon to adequately débride nonviable bone and soft tissues with less apprehension of massive bone defects [3, 8, 18, 21, 27, 34]. However, the prolonged use of an external fixator is a burden for patients with time-dependent incidence of complications [13, 26, 31]. The consolidation phase of distraction osteogenesis serves as a natural point of intervention to remove the external fixator, thus shortening the time patients are in the frame. However, the conversion to internal fixation must avoid any iatrogenic contamination and be strong enough to stabilize the immature bone (regenerate) [1, 9, 25, 28, 39, 41]. The onerous patient experience with traditional Ilizarov reconstruction is a powerful way to completely restore nearnormal anatomy and restoration of function in a cost-effective manner [23]. Improvement in the patient's treatment experience, through minimizing external fixator-related complications, should result in better patient outcomes; however, this needs to be supported by comparative studies. We compared the integrated fixation technique to the classic group in a uniform group of patients (bone defects of the tibia exclusively as a result from trauma) to elucidate if combining internal and external fixation is a safe way to expedite the time patients are in an external fixator.

This study had a number of limitations. First, this was a retrospective study and not randomized. Analysis of the patient demographics demonstrated a selection bias. In addition, the treatment strategy evolved over the study period; more recent surgeries had increased likelihood of 
integrated fixation techniques and specifically lengthening and then nailing. The study had strict inclusion criteria to which patients would be candidates for integrated fixation and therefore the method of Ilizarov reconstruction was not used for all comers. This study represents a best-case scenario of what can be expected with our limb reconstruction pathway. Future randomized studies would elucidate the ideal indications for the integrated technique. The patients treated in the classic group were more likely male and have a soft tissue flap, whereas those in the integrated fixation group were older. This represents the selection criteria used for the integrated technique in that presence of a large soft tissue defect precluded the integrated fixation and older patients were thought to be less tolerable for prolonged time in the external fixator. The small sample size and unique injury and patient characteristics did not allow for complete randomization; however, the total bone loss, presence of cigarette use, and infection were matched, arguing against any major invalidities in the methodology. Furthermore, older age has not been shown to significantly affect bone healing in nonunion surgery [38]. Second, because this study is a retrospective chart review, the final functional outcomes were recorded using what was found in the chart; however, no patients were lost to followup, the same senior surgeons (ATF, SRR) examined all patients, and the loss and inaccuracy of information should be small and distributed equally in both groups.

Third, the definition of bone healing to allow for removal of the external fixator and for full weightbearing after integrated fixation is subjective. This is consistent with the trauma and limb lengthening literature [6]. This represents an assessment bias because the senior surgeon who performed the procedure and who is motivated for the evolved surgical technique is also deciding when to remove the external fixator. In effect, he may have removed the external fixator earlier in patients in the integrated (new) procedure. We feel that this bias is not important. Both senior surgeons have a unique practice that solely focuses on complex extremity reconstruction using the Ilizarov method and have a combined 22 years of clinical experience. Premature removal of the external fixator is a serious and disruptive adverse event causing regenerate malunion or refracture. This requires reapplication of the frame and to salvage the reconstruction. Because there is still no objective radiographic finding for regenerate stability that allows the frame to be removed, it is an art that relies heavily on the clinical experience of the treating surgeons. Fourth, the integrated fixation group combined dissimilar interventions (insertion of a reamed intramedullary nail or application of a plate), which seem to have different effects on regenerate healing [33]. The general indications for use of insertion of a reamed intramedullary nail after deformity correction were closed physes and no active medullary infection. The general indications of lengthening and then insertion of a plate were a healed soft tissue envelope located at the intended internal fixation site and a periarticular deformity. The remainder of the patients were treated with the classic technique.

We found that patients treated with the integrated approach spent less time in the external fixator. Patients in the classic method of distraction osteogenesis required, on average, 11 months of treatment in the frame compared with 7 months treated with integrated fixation (Table 1). This finding of decreased time in an external fixator is similar to other series of lengthening and then nailing and lengthening and then plating techniques $[16,33]$. Furthermore, the external fixation index was also decreased in the integrated fixation group compared with the classic group, 61 days $/ \mathrm{cm}$ versus 75 days $/ \mathrm{cm}$, respectively. Previous studies did not perform integrated fixation techniques, making the comparison of external fixation index in the literature limited. The external fixation index for classic tibial Ilizarov reconstruction has ranged from 48 days $/ \mathrm{cm}$ to 75 days $/ \mathrm{cm}$ for a mean tibial defect of $4.7 \mathrm{~cm}$ to $6.5 \mathrm{~cm}[5,7,14]$.

The Ilizarov method of complex extremity reconstruction is accepted in the literature; however, the prolonged treatment time and patient-centered outcomes have questioned its use because patient-requested amputation has been documented as a result of intolerance of the treatment $[14,17,24,29,36,37]$. In the present study, no patient in either group requested an amputation; however, the impact on patients' impression of the treatment algorithm was not directly assessed in this study and should be an outcome measure to be analyzed in future studies. The mean bone defect in both groups was $5.3 \mathrm{~cm}$. Bone defect sizes exceeding $8 \mathrm{~cm}$ have been shown to increase complication rates [10,30]; although a regression analysis could not be performed with the data in the current study, a larger study may demonstrate more robust benefits for integrated fixation techniques.

Overall, there were 1.6 complications per patient. This is decreased compared with other studies of Ilizarov tibial reconstruction, which have rates that range between 2.1 and 2.6 per patient $[2,8,15,37]$. There was no difference in the number or severity of adverse event in both groups. Specifically, there was no late refracture in the integrated fixation group or any cases of intramedullary sepsis in those patients in the lengthening and then nailing subgroup. This is not surprising; although our study represents a challenging group of patients, the treatment algorithm is similar for all patients. Nonviable bone is resected, soft tissue defects are addressed at the index surgery, culture-specific antibiotics are administered for a 6-week duration by an infectious disease specialist, and initial stability and deformity correction are achieved with a Taylor Spatial Frame. This has 
been consistently performed over the study period by the senior surgeons (ATF, SRR) who adhered carefully to previously described integrated fixation techniques [16, 33]. The mounting of the proximal ring and a custom intramedullary nail that is short enough to avoid the distal points of fixation allow for a healthy medullary canal to serve as a physiologic boundary for infection. Overall, patients required a mean of 4.05 surgical procedures (range, $2-5$ procedures) for limb salvage. There was no difference in the incidence of unplanned surgical procedures (obstacles) between groups. Rates of additional surgeries to achieve union range from 2.2 to 3.5 ; however, the methods of Ilizarov limb reconstruction and patient populations differ among the published studies [14, 29, 35, 37].

The Association for the Study and Application of Methods of Ilizarov function and bone scores were good/excellent in $100 \%$ and $98 \%$ of patients, respectively. The distribution of good/excellent scores was similar in both groups. Importantly, there was a $100 \%$ union rate and no reinfection, especially in the integrated fixation group. Patients in both groups also did not demonstrate any refractures after removal of the external fixator. This is comparable to the experience of other authors. Sen et al [37] studied 24 patients with acute posttraumatic tibial bone loss. The mean bone defect was $5 \mathrm{~cm} ; 42 \%$ of patients had Type IIIB open fractures. All patients were treated with the classic method of lengthening and all patients achieved union. At a mean followup of 30 months, they reported $100 \%$ good/excellent Association for the Study and Application of Methods of Ilizarov bone and 96\% good/excellent Association for the Study and Application of Methods of Ilizarov function results [37]. Gulabi et al [15] reported on five patients treated with a lengthening over a nail technique for a mean $8.6-\mathrm{cm}$ tibial bone loss. This technique differs from the majority of integrated fixation protocols that were used in the present study; nonetheless, they achieved $100 \%$ union rates and no refractures. Association for the Study and Application of Methods of Ilizarov bone results were excellent in all five patients. Four patients reported excellent Association for the Study and Application of Methods of Ilizarov function results, whereas one patient was reported as good [15]. In a study of 28 patients with open tibial fractures, Atef and El-Tantawy [2] reported 16 excellent results, nine good, two fair, and one poor. The group was treated using circular fixation alone.

Patients in the integrated fixation group required weightbearing limitations from weightbearing as tolerated 30-lb toe-touch weightbearing when internal fixation was performed. Weightbearing was allowed typically after 6 weeks when the regenerate healed. One advantage of using an external fixator is that they are strong enough to allow patients to fully weightbear and maintain their functional status. In 28 patients $(48 \%)$ they were limited and potentially lost some of this functionality, although patients typically are able to perform more ROM exercises without the muscle-transfixing wires. These conflicting benefits have not been elucidated in the literature and should be looked at in future studies.

Limb salvage with distraction osteogenesis in the presence of posttraumatic tibial bone loss is a challenging surgical entity. In our series of 58 patients, there was a mean bone loss of $5.3 \mathrm{~cm}$ (range, 1.6-13 cm), 29 patients $(50 \%)$ of patients were actively infected, and 10 patients $(17 \%)$ were active smokers. The integrated fixation method allows for a more efficient limb salvage surgical reconstruction in patients carefully selected for that approach, whereas the frequency of adverse events and ability to restore limb lengths was not different between the groups with the numbers available. Careful placement of external fixation pins is critical to decrease cross-contamination with planned internal fixation constructs. In our study, good/excellent results were found in all patients after a mean of four surgical procedures; however, a larger multicenter prospective study would allow for more robust and generalizable conclusions.

Acknowledgments We thank Huong $\mathrm{Do} \mathrm{PhD}$, for her help with the statistical analysis.

\section{References}

1. Apivatthakakul T, Arpornchayanon O. Minimally invasive plate osteosynthesis (MIPO) combined with distraction osteogenesis in the treatment of bone defects. A new technique of bone transport: a report of two cases. Injury. 2002;33:460-465.

2. Atef A, El-Tantawy A. Management of open infected comminuted tibial fractures using Ilizarov concept. Eur J Orthop Surg Traumatol. 2014;24:403-408.

3. Cattaneo R, Catagni M, Johnson EE. The treatment of infected nonunions and segmental defects of the tibia by the methods of Ilizarov. Clin Orthop Relat Res. 1992;280:143-152.

4. Cierny G, Mader JT. Adult chronic osteomyelitis. Orthopedics. 1984;7:1557-1564.

5. Cierny G 3rd, Zorn KE. Segmental tibial defects. Comparing conventional and Ilizarov methodologies. Clin Orthop Relat Res. 1994;301:118-123.

6. Corrales LA, Morshed S, Bhandari M, Miclau T 3rd. Variability in the assessment of fracture-healing in orthopaedic trauma studies. J Bone Joint Surg Am. 2008;90:1862-1868.

7. Dagher F, Roukoz S. Compound tibial fractures with bone loss treated by the Ilizarov technique. J Bone Joint Surg Br. 1991;73:316-321.

8. Dendrinos GK, Kontos S, Lyritsis E. Use of the Ilizarov technique for treatment of non-union of the tibia associated with infection. J Bone Joint Surg Am. 1995;77:835-846.

9. Endo H, Asaumi K, Mitani S, Noda T, Minagawa H, Tetsunaga T, Ozaki T. The minimally invasive plate osteosynthesis (MIPO) technique with a locking compression plate for femoral lengthening. Acta Med Okayama. 2008;62:333-339.

10. Eralp L, Kocaoglu M, Polat G, Bas A, Dirican A, Azam ME. A comparison of external fixation alone or combined with 
intramedullary nailing in the treatment of segmental tibial defects. Acta Orthop Belg. 2012;78:652-659.

11. Fischgrund J, Paley D, Suter C. Variables affecting time to bone healing during limb lengthening. Clin Orthop Relat Res. 1994;301:31-37.

12. Gantsoudes GD, Fragomen AT, Rozbruch SR. Intraoperative measurement of mounting parameters for the Taylor Spatial Frame. J Orthop Trauma. 2010;24:258-262.

13. Ghoneem HF, Wright JG, Cole WG, Rang M. The Ilizarov method for correction of complex deformities. Psychological and functional outcomes. J Bone Joint Surg Am. 1996;78:1480-1485.

14. Green SA, Jackson JM, Wall DM, Marinow H, Ishkanian J. Management of segmental defects by the Ilizarov intercalary bone transport method. Clin Orthop Relat Res. 1992;280:136-142.

15. Gulabi D, Erdem M, Cecen GS, Avci CC, Saglam N, Saglam F. Ilizarov fixator combined with an intramedullary nail for tibial nonunions with bone loss: is it effective? Clin Orthop Relat Res. 2014;472:3892-3901.

16. Harbacheuski R, Fragomen AT, Rozbruch SR. Does lengthening and then plating (LAP) shorten duration of external fixation? Clin Orthop Relat Res. 2012;470:1771-1781.

17. Hutson JJ Jr, Dayicioglu D, Oeltjen JC, Panthaki ZJ, Armstrong MB. The treatment of gustilo grade IIIB tibia fractures with application of antibiotic spacer, flap, and sequential distraction osteogenesis. Ann Plast Surg. 2010;64:541-552.

18. Ilizarov GA. Clinical application of the tension-stress effect for limb lengthening. Clin Orthop Relat Res. 1990;250:8-26.

19. Kim SJ, Mandar A, Song SH, Song HR. Pitfalls of lengthening over an intramedullary nail in tibia: a consecutive case series. Arch Orthop Trauma Surg. 2012;132:185-191.

20. Kocaoglu M, Eralp L, Kilicoglu O, Burc H, Cakmak M. Complications encountered during lengthening over an intramedullary nail. J Bone Joint Surg Am. 2004;86:2406-2411.

21. Krappinger D, Irenberger A, Zegg M, Huber B. Treatment of large posttraumatic tibial bone defects using the Ilizarov method: a subjective outcome assessment. Arch Orthop Trauma Surg. 2013;133:789-795.

22. Kristiansen LP, Steen H. Lengthening of the tibia over an intramedullary nail, using the Ilizarov external fixator. Major complications and slow consolidation in 9 lengthenings. Acta Orthop Scand. 1999;70:271-274.

23. Lowenberg DW, Buntic RF, Buncke GM, Parrett BM. Long-term results and costs of muscle flap coverage with Ilizarov bone transport in lower limb salvage. J Orthop Trauma. 2013;27:576-581.

24. Maini L, Chadha M, Vishwanath J, Kapoor S, Mehtani A, Dhaon BK. The Ilizarov method in infected nonunion of fractures. Injury. 2000;31:509-517.

25. Oh CW, Shetty GM, Song HR, Kyung HS, Oh JK, Min WK, Lee BW, Park BC. Submuscular plating after distraction osteogenesis in children. J Pediatr Orthop B. 2008;17:265-269.

26. Paley D. Problems, obstacles, and complications of limb lengthening by the Ilizarov technique. Clin Orthop Relat Res. 1990;250:81-104.
27. Paley D, Catagni MA, Argnani F, Villa A, Benedetti GB, Cattaneo R. Ilizarov treatment of tibial nonunions with bone loss. Clin Orthop Relat Res. 1989;241:146-165.

28. Paley D, Herzenberg JE, Paremain G, Bhave A. Femoral lengthening over an intramedullary nail. A matched-case comparison with Ilizarov femoral lengthening. J Bone Joint Surg Am. 1997;79:1464-1480.

29. Paley D, Maar DC. Ilizarov bone transport treatment for tibial defects. J Orthop Trauma. 2000;14:76-85.

30. Papakostidis C, Bhandari M, Giannoudis PV. Distraction osteogenesis in the treatment of long bone defects of the lower limbs: effectiveness, complications and clinical results; a systematic review and meta-analysis. Bone Joint J. 2013;95:1673-1680.

31. Ramaker RR, Lagro SW, van Roermund PM, Sinnema G. The psychological and social functioning of 14 children and 12 adolescents after Ilizarov leg lengthening. Acta Orthop Scand. 2000;71:55-59.

32. Rozbruch SR, Fragomen AT, Ilizarov S. Correction of tibial deformity with use of the Ilizarov-Taylor spatial frame. $J$ Bone Joint Surg Am. 2006;88(Suppl 4):156-174.

33. Rozbruch SR, Kleinman D, Fragomen AT, Ilizarov S. Limb lengthening and then insertion of an intramedullary nail: a casematched comparison. Clin Orthop Relat Res. 2008;466:29232932.

34. Rozbruch SR, Pugsley JS, Fragomen AT, Ilizarov S. Repair of tibial nonunions and bone defects with the Taylor Spatial Frame. J Orthop Trauma. 2008;22:88-95.

35. Saleh M, Rees A. Bifocal surgery for deformity and bone loss after lower-limb fractures. Comparison of bone-transport and compression-distraction methods. $J$ Bone Joint Surg Br. 1995;77:429-434.

36. Sanders DW, Galpin RD, Hosseini M, MacLeod MD. Morbidity resulting from the treatment of tibial nonunion with the Ilizarov frame. Can J Surg. 2002;45:196-200.

37. Sen C, Kocaoglu M, Eralp L, Gulsen M, Cinar M. Bifocal compression-distraction in the acute treatment of grade III open tibia fractures with bone and soft-tissue loss: a report of 24 cases. J Orthop Trauma. 2004;18:150-157.

38. Taormina DP, Shulman BS, Karia R, Spitzer AB, Konda SR, Egol KA. Older age does not affect healing time and functional outcomes after fracture nonunion surgery. Geriatr Orthop Surg Rehabil. 2014;5:116-121.

39. Uysal M, Akpinar S, Cesur N, Hersekli MA, Tandogan RN. Plating after lengthening (PAL): technical notes and preliminary clinical experiences. Arch Orthop Trauma Surg. 2007;127:889893.

40. Watanabe K, Tsuchiya H, Sakurakichi K, Yamamoto N, Kabata $\mathrm{T}$, Tomita K. Tibial lengthening over an intramedullary nail. $J$ Orthop Sci. 2005;10:480-485.

41. Wu CC, Lee ZL, Wu CC, Lee ZL. Speeded gradual lengthening and secondary angled blade plate stabilisation for proximal tibial shaft non-union with shortening. Int Orthop. 2008;32:693-696. 